\title{
Transcription Driven Phase Separation in Chromatin Brush
}

\author{
Tetsuya Yamamoto* ${ }^{* \dagger}$ and Helmut Schiessel ${ }^{+}$ \\ ${ }^{\dagger}$ National Composite Center, Nagoya University Furo-cho, Chikusa-ku, Nagoya 464-8603, Japan \\ ${ }^{\ddagger}$ Instituut-Lorentz for Theoretical Physics, Niels Bohrweg 2, Leiden, NL-2333 CA, The Netherlands
}

Supporting Information

ABSTRACT: We theoretically predict the local density of nucleosomes on DNA brushes in a solution of molecules, which are necessary for transcription and the assembly of nucleosomes. Our theory predicts that in a confined space, DNA brushes show phase separation, where a region of relatively large nucleosomal occupancy coexists with a region of smaller nucleosomal occupancy. This phase separation is driven by an instability arising from the fact that the rate of transcription increases as the nucleosomal occupancy decreases due to the excluded volume interactions between nucleosomes and RNA polymerase during thermal diffusion

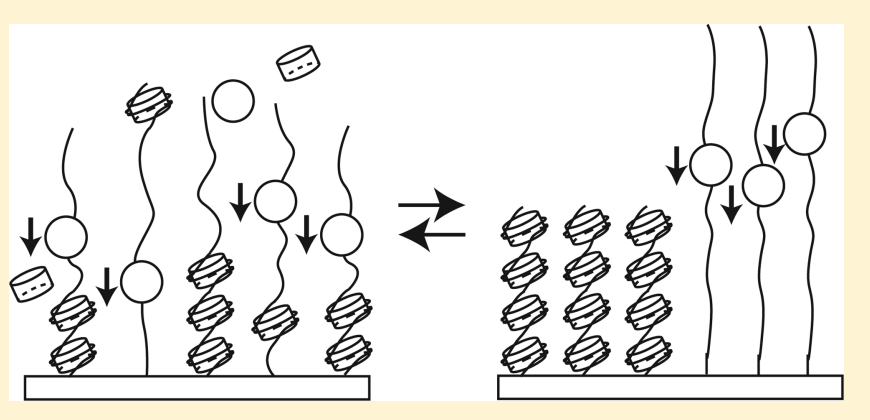
and, in turn, nucleosomes are (in some cases) desorbed from DNA when RNA polymerase collides with nucleosomes during transcription. The miscibility phase diagram shows critical points, which are sensitive to the rate constants involved in transcription, the changes of interactions of DNA chain segments by assembling nucleosomes, and pressures that are applied to the brushes.

\section{INTRODUCTION}

The DNA of eukaryotic cells is folded into compact chromatin structures to fit the size of their nuclei. ${ }^{1}$ The repeating unit of chromatin is the nucleosome, where DNA is wrapped around an octamer of histone proteins, in some cases guided by chaperons. The first step to express genetic information that is encoded in the sequence of nucleotides along DNA is transcription, by which the complementary sequence of RNA is synthesized by enzymes called RNA polymerase (RNAP). The rate of transcription of each gene decreases as the local packing density of nucleosomes at the corresponding sequence of DNA increases.

Recent experiments have shown that chromatin of embryonic stem (ES) cells displays fluctuations of the local nucleosome concentration over relatively long time $(\sim \min )$ and length scales $(\sim \mu \mathrm{m})$, analogous to critical fluctuations. ${ }^{2}$ In contrast, differentiated cells show more static structures, where regions of relatively large local concentrations of nucleosomes (heterochromatin) coexist with regions of relatively low local concentrations of nucleosomes (euchromatin), analogous to two-phase coexistent states. Because the transcription rate of each gene depends on the local concentrations of nucleosomes along the corresponding DNA stretch, the critical fluctuations of chromatin structures may play an important role in maintaining the pluripotency of ES cells. Moreover, the processes of phase separation during differentiation may be relevant to the lineage determination.

In the usual case of phase separation, critical fluctuations are driven by attractive interactions between molecules and random forces. ${ }^{3}$ Nucleosomes (which have net negative charges) show attractive interactions via histone tails (which have net positive charges), depending on the salt concentration. $^{4-7}$ At first glance, one may think that thermal fluctuations may play a role in random forces that destabilize the condensation of nucleosomes. However, nucleosomes are very stable structures that are rarely dissociated (in the sense that all their histone proteins are desorbed from DNA) by thermal fluctuations. ${ }^{8}$ Moreover, the thermal diffusion of nucleosomes along DNA is very slow, typically on the time scale of minutes to hours, even when the diffusion is not impeded by attractive interactions between nucleosomes. ${ }^{8-10}$ It is thus of interest to theoretically predict random forces that destabilize the condensed state of nucleosomes in stem cells.

Experiments have shown that, in some cases, histone proteins are desorbed from DNA when RNAP collides with nucleosomes during transcription. ${ }^{11,12}$ The collision between RNAP and nucleosomes may thus play a role in random forces that destabilize the condensed state of nucleosomes; the rate of transcription depends on the local concentrations of nuclesomes and transcription, in turn, remodels the structure of chromatin. The instability arising from this positive feedback may drive the critical fluctuations and the phase separation, which may be relevant to the dynamics of the chromatin structures of stem cells during development. The objective of this paper is to present a proof of this concept by using a simple system.

DNA brushes, in which DNA is end-grafted to a solid surface, are simple synthetic systems that enable us to measure the transcription rate while quantitatively changing the local

Received: February 4, 2016

Published: March 14, 2016 
packing density of DNA. ${ }^{13-17}$ When a DNA brush is set in a (dilute) solution of molecules that are necessary for transcription (such as RNAP) and the assembly of nucleosomes (such as histone proteins), one can also quantitatively control the local concentration of nucleosomes via the grafting density of DNA, the concentration of histone proteins in the solution, etc.

In this paper, we theoretically predict that DNA brushes show phase separation, where regions of relatively large local concentrations of nucleosomes coexist with regions of smaller concentrations of nucleosomes, when these brushes are confined (and thus the number of RNAP and histone proteins is fixed). This phase separation is driven by the instability arising from the fact that the transcription rate increases as the local concentrations of nucleosomes decreases (due to the excluded volume interactions between nucleosomes and RNAP during passive diffusion), and, in turn, the local concentrations of nucleosomes decreases as transcription rate increases (due to the collision between elongating RNAP and nucleosomes during transcription). Our theory also predicts that the critical point is sensitive to the rate constants involved in transcription, to the changes of interactions (from repulsive to attractive) between DNA chain segments by assembling nucleosomes, and to pressure that is applied to the brushes.

\section{MODEL}

2.1. Chromatin Brush. We take into account the dynamics of the assembly of nucleosomes and the desorption of their histone proteins from DNA chains in an extension of the model that has been used in ref 17. More formal treatment is shown in section S1 in the Supporting Information (SI). We treat a DNA brush, where DNA chains are end-grafted to a solid surface with grafting density $\sigma$ (see Figure 1). Each DNA chain is composed

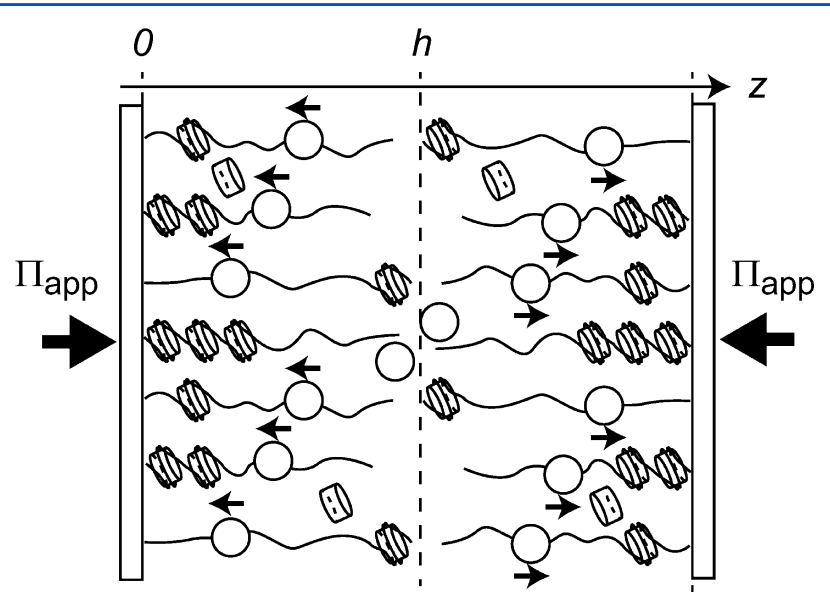

Figure 1. A model of a DNA brush, to which a pressure $\Pi_{\text {app }}$ is applied by pushing it against another brush. The space between the DNA chains of the two brushes is filled with a solution that includes RNAP (shown by spheres), histone proteins (shown by cylinders), and other molecules that are necessary for transcription and the assembly of nucleosomes.

of $N_{0}$ Kuhn segments of length $l_{\text {eff. }}$ The DNA brush is in a solution containing RNAPs and histones (and other small molecules that are necessary for transcription and the assembly of nucleosomes). Without changing the physics, we assume that each Kuhn segment has one binding site, which can be occupied by either a nucleosome or an RNAP, although, in reality, a Kuhn length of unoccupied DNA is $100 \mathrm{~nm}(\sim 294$ bp) at physiological salt concentration and is long enough to accommodate two nucleosomes, where DNA of length $147 \mathrm{bp}$ $(\sim 50 \mathrm{~nm})$ is the wrapping length per nucleosome. A nucleosome is a disk-shaped complex of diameter $10 \mathrm{~nm}$ and height $6 \mathrm{~nm}$, and DNA wraps around it by 1.65 turns. ${ }^{18,19}$ The length $l_{\text {eff }}$ of a Kuhn segment thus depends on whether this segment is occupied by a nucleosome or not (see also the treatment below eq 13).

We here treat a brush of DNA chains that are much longer than the Kuhn length of DNA, and the negative charges of these DNA chains are almost fully neutralized by salt in the solution. $6,7,16,20$ Although the distribution of DNA chain segments in DNA brushes has not been fully resolved, ${ }^{16,17}$ we here use the Alexander approximation, which assumes that the local concentration of Kuhn segments is uniform in the brush region. ${ }^{21}$ This treatment highlights the roles played by transcription for chromatin structures, rather than the details of brush models. With this approximation, the free ends of the DNA chains in the brush are located at the top of the brush and the grafted ends of the DNA chains are located at the bottom of the brush. For simplicity, we treat a DNA brush, where each of constituent DNA chains has a promoter at its free end and a terminator at its grafted end. The promoter and terminator regions are not occupied by nucleosomes due to their specific sequences (there is indeed nucleosome depletion at the transcription start and end sites $^{22}$ ). We use the Cartesian coordinate system, where $z$ is the distance from the solid surface (see Figure 1). An estimate of the orders of magnitude of parameters used in our theory are shown in Table 1.

Table 1. Orders of Magnitude of Parameters That Are Used in Our Theory Estimated for Physiologically Relevant Salt Concentration $^{a}$

$\begin{array}{ccc}\text { symbol } & \text { physical meaning } & \begin{array}{c}\text { orders of } \\ \text { magnitude }\end{array} \\ w_{\text {on }} & \text { second virial coefficient for interactions between } \\ \text { nucleosomes } & -1 \times 10^{6} \mathrm{~nm}^{3} \\ w_{\text {off }} & \text { second virial coefficient for interactions between } \\ \text { vacant DNA } & 3 \times 10^{4} \mathrm{~nm}^{3} \\ l_{\mathrm{a}} & \text { Kuhn length of vacant DNA } & 100 \mathrm{~nm} \\ \gamma & \text { Kuhn length change by nucleosomal assembly } & 0.9 \\ k_{\text {on }}^{\text {his }} & \text { rate constant of nucleosomal assembly } & 6.0 \times 10^{3} \\ & & \mathrm{M}^{-1} \mathrm{~s}^{-1}\end{array}$

${ }^{a}$ The value of $w_{\text {on }}$ that is predicted by using molecular dynamics simulations for a physiological salt concentration is shown here. ${ }^{4} \mathrm{An}$ experiment has determined a much smaller value $-1.5 \times 10^{3} \mathrm{~nm}^{3}$, but for a relatively small salt concentration $75 \mathrm{mM}^{6}$ The dimension of a DNA chain segment (the Kuhn lengh $l_{\mathrm{a}}$ is $100 \mathrm{~nm}$ and the diameter $d$ is $2 \mathrm{~nm}$ ) is used to estimate the value of $w_{\text {off. }}$ It is in agreement with an experimentally determined value $1 \times 10^{4} \mathrm{~nm}^{3}$ for the salt concentration $100 \mathrm{mM}^{23}$ For a more precise estimate, one should take into account the fact that a Kuhn segment can accommodate a maximum of two nucleosomes. The rate constant $k_{\text {on }}^{\text {his }}$ for cases in which the assembly of nucleosomes is guided by NAP1 chaperon is shown. $^{24}$

2.2. Unidirectional Motion of RNAP during Transcription. Transcription is initiated when an RNAP binds to a noncoding DNA sequence, called the promoter, by specific interactions and changes its conformation. The enzyme then moves unidirectionally toward another noncoding sequence, called the terminator, base-by-base, while synthesizing a chain of RNA. When the RNAP reaches the terminator, it is released 
from the DNA. We here treat the unidirectional motion of RNAP along the DNA of the brush in a steady state.

For cases in which the occupancy of RNAP on DNA chain segments is relatively small (and thus the RNAPs do not produce a traffic jam on the DNA chains), the binding rate $R_{\mathrm{p}}$ of RNAP to the promoter of a DNA chain (due to specific interactions) has the form

$$
R_{\mathrm{p}}^{\mathrm{rnp}}=\lambda \rho(h)
$$

The rate constant $\lambda$ accounts for the binding of RNAP to the promoter. $\rho(z)$ is the local concentration of RNAP in the solution of the brush region (which is derived by using eq 7 shown later), and $h$ is the height of the brush (see Figure 1). We neglected the desorption rate of RNAP from the promoters because RNAP firmly grips DNA chains once it changes its conformation.

In a steady state, the elongation rate $j_{\text {rnp }}$, with which RNAP unidirectionally moves from one binding site to the next along a DNA chain, does not depend on the position of the binding site. The elongation rate $j_{\text {rnp }}$ at a binding site has the form

$$
j_{\mathrm{e}}^{\mathrm{rnp}}=\xi n_{\text {rnp }}\left(1-n_{\text {his }}\right)
$$

where $\xi$ is the rate constant that accounts for the unidirectional motion of RNAP from a binding site to the next. The rate constant $\xi$ is (the ensemble average of) the inverse of the total time, with which an RNAP moves by a Kuhn length ( 294 bp), and we thus assume that this rate constant does not depend on the position of the binding site along a DNA chain. $n_{\text {rnp }}$ is the occupancy of RNAP at the binding site and $n_{\text {his }}$ is the occupancy of nucleosomes at the next binding site. The occupancies, $n_{\text {his }}$ and $n_{\text {rnp }}$, do not depend on the position of the binding site either (except for the promoter and terminator sites) due to the Alexander approximation (see also eq 11). The factor $1-n_{\text {his }}$ in eq 2 thus represents the fact that the motion of RNAP is suppressed when the next site is occupied by a nucleosome. RNAP is a processive motor, and thus once RNAP binds to the promoter, it does not desorb from the DNA chain before it reaches the terminator.

The desorption rate $R_{\mathrm{t}}$ of RNAP from the terminator of a DNA chain has the form

$$
R_{\mathrm{t}}^{\mathrm{rnp}}=k_{\mathrm{t}} n_{\mathrm{rnp}}\left(s_{\mathrm{t}}\right)
$$

where $k_{\mathrm{t}}$ is the rate constant that accounts for the release of RNAP from the terminator and $n_{\text {rnp }}\left(s_{t}\right)$ is the occupancy of RNAP at the terminator. The continuity of the fluxes of RNAP along DNA chains leads to the form

$$
R_{\mathrm{p}}^{\mathrm{rnp}}=j_{\mathrm{e}}^{\mathrm{rnp}}=R_{\mathrm{t}}^{\mathrm{rnp}}
$$

2.3. Diffusion of RNA Polymerase in Solution. To the solution in the brush region, the terminators and promoters act as the sources and sinks of RNAP, respectively. RNAP is released at the bottom of the brush (where the terminator regions are located) with rate $\sigma R_{\mathrm{t}}^{\text {rnp }}$ (per unit area) and adsorbed at the top of the brush (where the promoter regions are located) with the same rate $\sigma R_{\mathrm{p}}^{\text {rnp }}$ (per unit area) (see Section 2.2). The gradient of RNAP concentration is thus generated by the unidirectional motion of RNAP during transcription. ${ }^{13,14,17}$

The fluxes of RNAP arising from the concentration gradient has the form

$$
J_{\mathrm{rnp}}=-D_{\mathrm{rnp}}\left[\frac{\partial}{\partial z} \rho(z)+v \rho(z) \frac{\partial}{\partial z} \Phi_{\mathrm{on}}(z)\right]
$$

where the first term is the flux due to the thermal diffusion and the second term is the flux due to the nonspecific interactions between nucleosomes and RNAP. The derivation of eq 5 is shown in section S2 of the SI. $D_{\text {rnp }}$ is the diffusion constant of RNAP. $\Phi_{\text {on }}(z)$ is the local concentrations of nucleosomes, and $v$ is the second virial coefficient that accounts for the nonspecific interactions between nucleosomes and RNAP in the solution. Without changing the physics, we neglect the nonspecific interactions between RNAP and vacant DNA chain segments (that are not occupied by either RNAP or nucleosomes) in the solution. We treat cases in which the local concentration of RNAP in the solution of the brush region is small and thus the (RNAP)-(RNAP) interactions and the (RNAP)-(histone) interactions are negligible. Here and after, we write nonspecific interactions between component $\mathrm{A}$ and $\mathrm{B}$ as (A)-(B) interactions, for simplicity. The fluxes $J_{\text {rnp }}$ are constant in the brush (except for the top and bottom of the brush, where the promoter and terminator regions are located).

Because RNAP does not penetrate the solid surface, the fluxes of RNAP are zero at the bottom of the brush. This leads to the fact that the fluxes $J_{\text {rnp }}$ of RNAP in the solution are equal to the releasing rate of RNAP at the bottom of the brush,

$$
J_{\text {rnp }}=\sigma R_{\mathrm{t}}^{\mathrm{rnp}}
$$

The (net) fluxes $J_{\text {rnp }}$ of RNAP are also zero at the top of the brush because the number of RNAP in the brush region is constant in steady states. It is consistent with the fact that the fluxes $J_{\text {rnp }}$ of RNAP in the solution are equal to the adsorbing rate of RNAP (per unit area) at the top of the brush (see eqs 4 and 6). With the local equilibrium approximation, chemical potentials are continuous across the interface between the bulk solution and the solution in the brush region. This leads to the local concentration of RNAP at the top of the brush in the form

$$
\rho(h)=\rho_{0} \mathrm{e}^{-v \Phi_{\mathrm{on}}(h)}
$$

The derivation of eq 7 is shown in section S2 in the SI. With eq 7 , we assume that although the promoters of DNA chains are located at their free ends, the local concentration $\Phi_{\text {on }}(h)$ of RNAP at the vicinity of the promoters is smaller than the concentration of the bulk solution due to the excluded volume interactions between RNAP and nucleosomes. Experimentally, this may be effective for the cases that there is a small distance between the free ends of DNA chains and their promoter regions.

Because DNA chain segments are uniformly distributed in the DNA brush, the local concentrations of nucleosomes and vacant DNA chain segments in the solution of the brush region have the forms

$$
\begin{aligned}
& \Phi_{\text {on }}(z)=\frac{\sigma N_{0}}{h} n_{\text {his }} \\
& \Phi_{\text {off }}(z)=\frac{\sigma N_{0}}{h}\left(1-n_{\text {his }}\right)
\end{aligned}
$$

respectively. The interactions between RNAP and nucleosomes thus do not drive the fluxes of RNAP (see eq 5), and the local concentrations of RNAP in the brush region depend on the local concentrations $\Phi_{\text {on }}$ of nucleosomes only via the boundary condition (eq 7). With this boundary condition, the local 
concentration of RNAP in the solution of the brush region has the form

$$
\rho(z)=\rho(h)\left[1+\frac{\lambda \sigma}{D_{\mathrm{rnp}}}(h-z)\right]
$$

2.4. Assembly and Disassembly of Nucleosomes. In a steady state, the assembling rate of nucleosomes to a binding site of a DNA chain is equal to the desorption rate of the histone proteins of nucleosomes at the binding site. This equality reads

$$
k_{\text {on }}^{\text {his }} c\left(1-n_{\text {his }}\right)-\zeta n_{\text {rnp }} n_{\text {his }}=0
$$

The first term of eq 11 is the assembling rate of nucleosomes, and $k_{\mathrm{on}}^{\text {his }}$ is the rate constant that accounts for this process. $c$ is the local concentration of histone proteins in the solution in the brush region at the position of the binding site. The second term of eq 11 represents the fact that histone proteins are desorbed from DNA when RNAP collides with nucleosomes during transcription, and $\zeta$ is the rate constant that accounts for this process. With eq 11 , we neglected the spontaneous desorption of nucleosomes from DNA due to thermal excitation because it is a very slow process; relatively large free energy costs $\left(>15 k_{B} T\right)$ are necessary to desorb nucleosomes even when the dissociation is not suppressed by the attractive interactions between nucleosomes. ${ }^{8}$

In contrast to RNAP, histone proteins do not show unidirectional motion along a DNA chain and thus histone proteins are distributed uniformly in the solution of the brush region (in principle, one can think of a process, where RNAP pushes nucleosomes forward during a transcription, ${ }^{25}$ but we do not treat this process in this paper). The size of histone proteins is much smaller than the size of RNAP, ${ }^{11}$ and we thus neglect the (histone)-(nucleosome) interactions and the (histone)-(vacant DNA segment) interactions, for simplicity. We treat the cases that the local concentration of histone proteins in the solution of the brush region is relatively small, where the (histone)-(histone) interactions and the (RNAP)(histone) interactions are negligible. With this approximation, the concentration $c$ of histone proteins in the solution of the brush region is equal to the concentration $c_{0}$ of these proteins in the bulk solution.

We neglected a couple of molecular details involved in the assembly of nucleosomes: (i) the fact that nucleosomes are assembled from eight histone proteins (and thus the assembly rate is $\sim c_{0}^{8}$ in a more precise treatment), (ii) the specific chemistry of four types of core histone proteins (H2A, H2B, $\mathrm{H} 3$, and H4), and (iii) the fact that the assembly of nucleosomes is guided by chaperones. With the treatment (ii), four different types of histone proteins are treated as one type of molecule via the parameter $c_{0}$ for the cases that the solution includes $\mathrm{H} 2 \mathrm{~A}, \mathrm{H} 2 \mathrm{~B}, \mathrm{H} 3$, and $\mathrm{H} 4$ with equal concentrations.

2.5. Force Balance Equation of a Chromatin Brush. For the cases that the dynamics of the conformation of DNA chains is faster than the other processes, the height $h$ of a DNA brush is derived by minimizing the free energy (per unit area) that has the form

$$
f=f_{\text {pol }}+f_{\text {int }}+\Pi_{\text {app }} h
$$

with respect to the brush height $h . f_{\mathrm{pol}}$ is the free energy due to the entropic elasticity of DNA chains, $f_{\text {int }}$ is the free energy due to nonspecific interactions, and the third term is the work done by an applied pressure $\Pi_{\text {app. }}$. In this paper, we treat two cases: (i) a DNA brush alone in a solution, $\Pi_{\text {app }}=0$, and (ii) a DNA brush pushed against another DNA brush with applied pressures $\Pi_{\text {app }}(>0)$ (see Figure 1). In the case (ii), the functional form of the free energy for the two brushes is identical (although the values of the free energy may be different in some cases) and thus the free energy of the system is the sum of the free energies of the two brushes.

The free energy $f_{\text {pol }}$ due to the entropic elasticity of DNA chains has the form

$$
\frac{f_{\mathrm{pol}}}{T}=\frac{3}{2} \frac{\sigma h^{2}}{N_{0} l_{\mathrm{eff}}^{2}}
$$

where $T$ is the absolute temperature in units of the Boltzmann constant. We use the form $l_{\text {eff }}=l_{\mathrm{a}}\left(1-\gamma n_{\text {his }}\right)$ to treat the effective length $l_{\text {eff }}$ of chain segments, where the constant $\gamma>0$ accounts for the fact that the length of DNA chain segments becomes shorter when they are reeled around histone proteins.

The free energy $f_{\text {int }}$ due to nonspecific interactions has the form

$$
\begin{aligned}
\frac{f_{\text {int }}}{T}= & \int \mathrm{d} z\left[\frac{1}{2} w_{\text {on }} \Phi_{\text {on }}^{2}(z)+w_{\text {int }} \Phi_{\text {on }}(z) \Phi_{\text {off }}(z)\right. \\
& \left.+\frac{1}{2} w_{\text {off }} \Phi_{\text {off }}^{2}(z)\right]+\frac{1}{3} u \int \mathrm{d} z \Phi_{\text {on }}^{3}(z)
\end{aligned}
$$

The second virial coefficients $w_{\text {on }}, w_{\text {int }}$ and $w_{\text {off }}$ account for the (nucleosome)-(nucleosome) interactions, the (nucleosome)(vacant DNA segment) interactions, and the (vacant DNA segment)-(vacant DNA segment) interactions, respectively; the interactions between DNA chain segments change from repulsive to attractive when nucleosomes are assembled at the DNA chain segments ${ }^{4-6}$ (see also Table 1$), u$ is the third virial coefficient that accounts for the three-body interactions between nucleosomes. Here we treat the cases in which the local concentrations of RNAP and histone are relatively small in the solution of the brush region and thus neglected the interactions between these proteins and DNA chain segments. For simplicity, we do not explicitly treat the fact that nucleosomes, due to their attractive interactions, assemble into chromatin fibers.

Minimizing the free energy, eq 12, with respect to the brush height $h$ leads to the force balance equation that has the form

$$
\begin{aligned}
-\frac{\Pi_{\mathrm{app}}}{T}= & \frac{3 \sigma h}{N_{0} l_{\mathrm{eff}}^{2}}-\frac{w \sigma^{2} N_{0}^{2}}{2 h^{2}}\left(n_{\mathrm{his}}-n_{+}\right)\left(n_{\mathrm{his}}-n_{-}\right) \\
& -\frac{2}{3} u \frac{\sigma^{3} N_{0}^{3}}{h^{3}}
\end{aligned}
$$

with $w=w_{\text {on }}+w_{\text {off }}-2 w_{\text {int }}$ and $n_{ \pm}=\left(w_{\text {off }}-w_{\text {int }} \pm \sqrt{w_{\text {int }}^{2}-w_{\text {on }} w_{\text {off }}}\right) / w$. We derived the third term of eq 15 by using the fact that this term is only significant for $n_{\text {his }} \simeq 1$. The first and second terms of eq 15 are the contributions of the entropic elasticity and the two-body interactions between DNA chain segments to the osmotic pressure of the brush. These terms define the scales of the height and osmotic pressure of the Alexander brush in the forms 


$$
\begin{aligned}
& h_{\mathrm{Alx}}=N_{0} l_{\mathrm{a}}\left(\frac{w \sigma}{6 l_{\mathrm{a}}}\right)^{1 / 3} \\
& \Pi_{\mathrm{Alx}}=\frac{3 \sigma \mathrm{T}}{N_{0} l_{\mathrm{a}}} h_{\mathrm{Alx}}
\end{aligned}
$$

where $h_{\mathrm{Alx}}$ is the height of the Alexander brush and $\Pi_{\mathrm{Alx}}$ is the applied pressure to compress the brush by $\sim 1 / 4$ of the height $h_{\mathrm{Alx}}$.

2.6. Nucleosomal Occuapncy in a Steady State. Solving eqs 4 and 11 leads to the occupancies, $n_{\text {rnp }}$ and $n_{\text {his }}$ in the forms

$$
\begin{aligned}
& n_{\mathrm{rnp}}=\frac{\lambda \rho(h)}{2 \xi}\left(1+\sqrt{1+\frac{4}{\eta}}\right) \\
& n_{\mathrm{his}}=\frac{1}{2} \eta\left(1+\frac{2}{\eta}-\sqrt{1+\frac{4}{\eta}}\right)
\end{aligned}
$$

with

$$
\eta=\frac{\lambda \rho(h) \zeta}{k_{\mathrm{on}}^{\text {his }} c_{0} \xi}
$$

The local concentration $\rho(h)$ of RNAP at the positions of the promoters depends on the occupancy $n_{\text {his }}$ of nucleosomes and the height $h$ of the brush (see eq 7). We first derive the brush height as a function of the occupancy $n_{\text {his }}$ by using eq 15 . By using this function, eq 19 is now a self-consistent equation only of the occupancy $n_{\text {his }}$ of nucleosomes. This self-consistent equation has maximal three solutions, and the stability of these solutions is analyzed by the linear stability analysis (see section S3 in the SI). We use the self-consistent equation to derive the occupancy $n_{\text {his }}$ of nucleosomes as functions of the rate constants that are relevant to transcription, the extent of the changes of interactions between DNA chain segments by assembling nucleosomes, and applied pressures.

\section{RESULTS}

3.1. A DNA Brush in a Bulk Solution. We first treat cases in which a DNA brush is alone in a bulk solution, $\Pi_{\text {app }}=0$. This solution plays the role of the reservoir of RNAP and histone proteins (and other molecules that are necessary for transcription and the assembly of nucleosomes).

When the concentration $\rho_{0}$ of RNAP in the bulk solution is very small, DNA chains in the brush are fully occupied by nucleosomes and the brush is collapsed (see Figure 2). For the cases that the parameter $n_{-}$(which is the combination of the second virial coefficients; see below eq 15) is smaller than a critical value $n_{-}^{\mathrm{c}}$, the occupancy $n_{\text {his }}$ of nucleosomes decreases only slightly with increasing the rescaled rate constant $\eta_{0}$ (三 $\left.\lambda \rho_{0} \zeta /\left(k_{\text {on }}^{\text {his }} c_{0} \xi\right)\right)$ that is relevant to transcription until this rescaled rate constant becomes larger than a threshold value $\eta_{\text {oth }}$ (see the red curve in Figure $2 \mathrm{a}$ ). At the threshold value $\eta_{0 \text { th }}$ of the rescaled rate constant, the occupancy $n_{\text {his }}$ jumps to a smaller value, and the brush height $h$ jumps to a larger value. This jump is analogous to first order phase transitions. For larger values of the rescaled rate constant $\eta_{0}$, the occupancy $n_{\text {his }}$ decreases continuously with increasing the constant $\eta_{0}$.

The binding transition is driven by an instability arising from two types of interactions between nucleosomes and RNAP: (1) The local concentrations of nucleosomes decrease as the transcription rate increases because histone proteins are desorbed from DNA chains due to the collision between a.

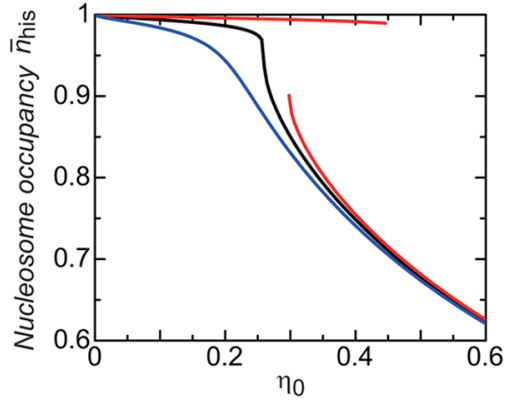

b.

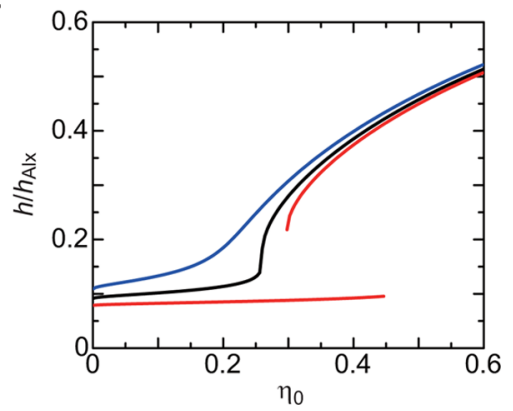

Figure 2. (a) The occupancy $n_{\text {his }}$ of nucleosomes and (b) the rescaled height $h / h_{\text {Alx }}$ of a DNA brush in the bulk solution is shown as functions of the rescaled rate constant $\eta_{0}\left(\equiv \lambda \rho_{0} \zeta /\left(k_{\mathrm{on}}^{\text {his }} c_{0} \xi\right)\right)$ for the cases that the values of the combination $n_{-}$of the second virial coefficients (defined below eq 15) are 0.975 (red), 0.983363 (black), and 0.995 (blue). The values of parameters that are used for the calculations are $n_{+}=1.8, \gamma=0.7, v \sigma N_{0} / h_{\mathrm{Alx}}=0.8$, and $4 u \sigma N_{0} /\left(3 w h_{\mathrm{Alx}}\right)$ $=2.0 \times 10^{-3}$. $h_{\mathrm{Alx}}$ is the length scale of the brush height, which is defined by eq 16 .

nucleosomes and RNAP during transcription. (2) In turn, as the local concentrations of nucleosomes decrease, the excluded volume interactions between nucleosomes and RNAP in the solution decrease and thus RNA polymerase is recruited to the brush region; this increases the transcription rate in the DNA brush.

The jump of the nucleosomal occupancy at the threshold rate constant $\eta_{\text {th }}$ decreases with increasing the combination $n_{-}$ of the second virial coefficients, and eventually, the binding transition becomes continuous for $n_{-}=n_{-}^{c}$, (see the black curve in Figure 2). The continuous binding transition is analogous to second-order phase transitions. For larger values of the parameter $n_{-}$, the nucleosomal occupany decreases continuously with increasing the rescaled rate constant $\eta_{0}$, and the DNA brush does not show binding transitions (see the blue curve in Figure 2). The nature of the binding transition is thus sensitive to the rescaled rate constant $\eta_{0}$ and the combination $n_{-}$of the second virial coefficients.

3.2. Binding Transitions Driven by Applied Pressures. The brush height $h$ jumps at the binding transitions (see Figure 2b). This implies that binding transitions may also be driven by applying pressures to a DNA brush. Here we treat the case that a pressure is applied to a DNA brush by pushing it against another DNA brush.

When the rescaled rate constant $\eta_{0}$ is larger than a critical value $\eta_{0 c}$ the height of the brush shows a jump to a smaller value at a threshold pressure (see the magenta and cyan curves in Figure $3 b$ ). The occupancy $n_{\text {his }}$ of nucleosomes also shows a jump at the threshold pressure (see the magenta and cyan curves in Figure 3a). The transition of the brush height is thus driven by the instability arising from the two types of the 


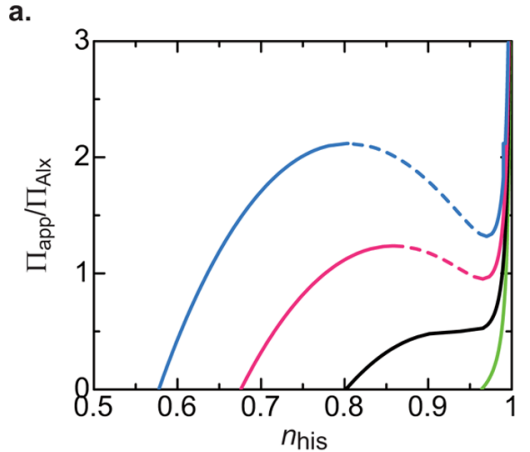

b.

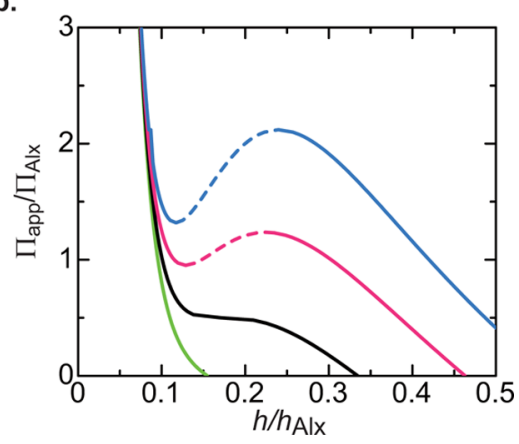

Figure 3. (a) The occupancy $n_{\text {his }}$ of nucleosomes and (b) the (rescaled) height $h / h_{\mathrm{Alx}}$ of a chromatin brush as functions of (rescaled) applied pressures $\Pi_{\text {app }} / \Pi_{\mathrm{Alx}}$ for the cases that the values of the rescaled rate constant $\eta_{0}\left(\equiv \lambda \rho_{0} \zeta /\left(k_{\text {on }}^{\text {his }} c_{0} \xi\right)\right)$ are 0.2 (green), 0.33443 (black), 0.5 (magenta), and 0.7 (cyan). $h_{\mathrm{Alx}}$ and $\Pi_{\mathrm{Alx}}$ are the scales of the brush height and osmotic pressure (defined by eqs 16 and 17). The broken curves show unstable solutions (determined by the linear stability analysis in section S3 in the SI). The values of parameters that are used for the calculations are $n_{+}=1.8, n_{-}=0.99, \gamma=0.7, v N_{0} \sigma / h_{\mathrm{Alx}}=0.8$, and $4 u \sigma N_{0} /\left(3 w h_{\mathrm{Alx}}\right)=2.0 \times 10^{-3}$.

interactions between nucleosomes and RNAP, analogous to the cases shown in section 3.1. The jump of the brush height decreases with decreasing the rescaled rate constant $\eta_{0}$, and, at the critical rescaled rate constant $\eta_{00}$ the transition of the brush height becomes a continuous transition, analogous to the second order phase transitions (see the black curve in Figure 3 ). For smaller values of the rescaled rate constant, the brush height decreases continuously with increasing applied pressures without showing binding transitions. These results are summarized in a phase diagram (see Figure 4).

3.3. Phase Separation of Chromatin Brushes. Thus, far, we have treated the binding transitions of chromatin brushes in the bulk solutions, which serve as the reservoir of RNAP and histone proteins. In a biological system, DNA chains, RNAP, and histone proteins are confined in the interior of a nucleus and thus the number of RNAP and histone proteins is fixed. Because of the confinement, chromatin in a nucleus may show phase separation, where collapsed chromatin regions of relatively large nucleosomal occupancy coexists with expanded chromatin regions of smaller nucleosomal occupancy. Here we treat the cases that RNAP and histone proteins are confined in a finite space with two interacting DNA brushes, analogous to the situation of chromatin in a nucleus. We assume that other small molecules that are necessary for transcription and the assembly of nucleosomes are abundant in the system. In an experiment on a synthetic system, ribonuclease may be necessary to keep the concentration of NTP constant and suppress the side-effects arising from the RNA products.

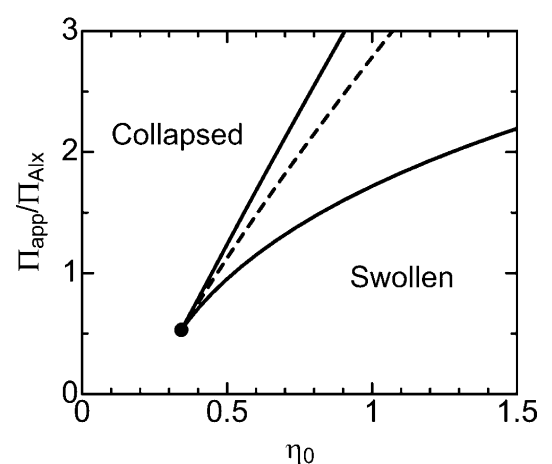

Figure 4. Phase diagram of a chromatin brush is shown in the parameter space of rescaled rate constant $\eta_{0}\left(\equiv \lambda \rho_{0} \zeta /\left(k_{\mathrm{on}}^{\text {his }} c_{0} \xi\right)\right)$ and rescaled applied pressures $\Pi_{\mathrm{app}} / \Pi_{\mathrm{Alx}}$. The solid curve is the boundary between the swollen state and the collapsed state, and the state in the region that is enclosed by these solid curves depends on the history of applied pressures (see Figure 3). The broken curve is determined by using the Maxwell construction to Figure $3 \mathrm{~b}$. These solid curves end at the critical point, $\eta_{0 \mathrm{c}}=0.33443$ and $\Pi_{\mathrm{c}} / \Pi_{\mathrm{Alx}}=0.500539$. The values of parameters that are used for the calculations are $n_{+}=1.8, n_{-}=0.99, \gamma=$ $0.7, v N_{0} \sigma / h_{\mathrm{Alx}}=0.8$, and $4 u \sigma N_{0} /\left(3 w h_{\mathrm{Alx}}\right)=2.0 \times 10^{-3} \cdot h_{\mathrm{Alx}}$ and $\Pi_{\mathrm{Alx}}$ are the scales of the height and osmotic pressures of polymer brushes (defined by eqs 16 and 17).

In contrast to the usual cases of the phase separation of (chemically end-grafted) multicomponent polymer brushes, ${ }^{26-30}$ in principle, DNA brushes can show macroscopic (lateral) phase separation because the occupancy of nucleosomes is not constrained by the fact that DNA chains are endgrafted to a surface. In this section, we thus predict the macroscopic phase separation of DNA brushes. The possibility of microphase separation and the patterns of domains due to the phase separation will be discussed in section 4 .

The chemical potentials, $\mu_{\text {rnp }}$ and $\mu_{\text {his }}$ of RNAP and histone proteins are continuous across the top of the two brushes. These chemical potentials are the Lagrange multipliers that ensure that the number of RNAP and histone proteins that are initially included in the system is constant. For simplicity, we here use these chemical potentials to represent the initial number of RNAP and histone proteins, although it is somewhat indirect. The spinodal curves are derived by using the condition, at which two branches of the solutions show instability (see section S3 in the SI for the details of the derivation). A uniform chromatin brush is unstable in the region that is enclosed by the spinodal curves; see the broken curves in Figure 5. It is not straightforward to predict the binodal curves because the two coexisting phases are both nonequilibrium steady states. Here we tentatively derive the binodal curves by using the Maxwell construction to the $\Pi_{\text {app }}-$ $h$ curves (see Figure S1 in the SI). We discuss the validity of this treatment in section 4.

Our theory predicts that DNA brushes show phase separation in the region that is enclosed by the binodal curves (see the solid curves in Figure 5). This theory also predicts that the regions of two-phase coexistent states have a critical point. This phase separation is driven by the type of instability, which is essentially the same as the binding transitions of a DNA brush in a bulk solution: RNAP tends to localize in the regions of smaller nucleosomal concentrations due to the excluded volume interactions between nucleosomes and RNAP in the solution. This enhances the transcription rate in these regions. The concentrations of nucleosomes in these regions decrease 
a.

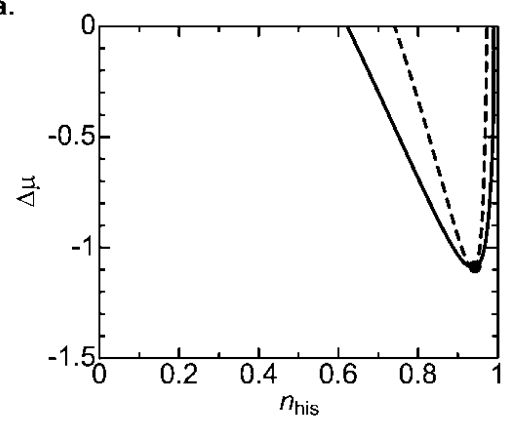

b.

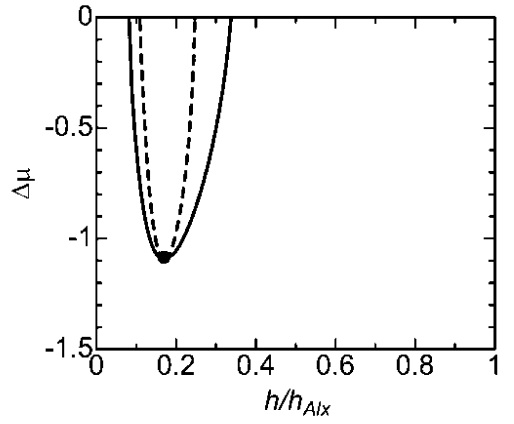

c.

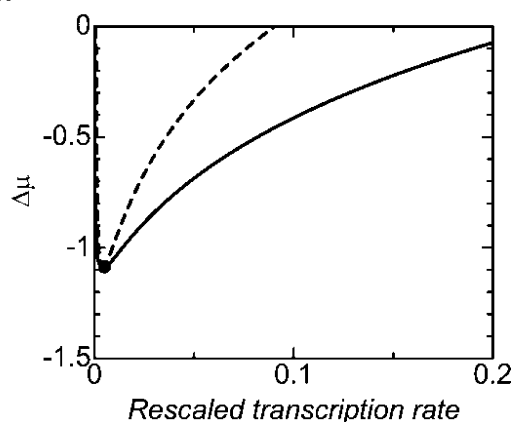

Figure 5. (a) The occupancy $n_{\text {his }}$ of nucleosomes, (b) the height, and (c) the rescaled transcription rate $\eta$ (see eq 20) of the two coexisting regions of a DNA brush is shown as functions of $\Delta \mu\left(\equiv \mu_{\mathrm{rnp}}-\mu_{\text {his }}+\right.$ $\left.\log \left(\lambda \zeta /\left(k_{\mathrm{on}}^{\text {his }} \xi\right)\right)\right)$, which is a linear function of the difference between the chemical potentials $\mu_{\text {rnp }}$ of RNAP and the chemical potentials $\mu_{\text {his }}$ of histone proteins. The solid curves are binodal curves that are predicted by using the Maxwell construction to $\Pi_{\text {app }}-h$ curves, and the broken curves are spinodal curves. The critical point is highlighed by the filled circle. The values of parameters that are used for the calculations are $n_{+}=1.8, n_{-}=0.99, \gamma=0.7, v N_{0} \sigma / h_{\mathrm{Alx}}=0.8$, and $4 u \sigma N_{0} /\left(3 w h_{\mathrm{Alx}}\right)=2.0 \times 10^{-3} \cdot h_{\mathrm{Alx}}\left(\equiv N_{0} l_{\mathrm{a}}\left(w \sigma /\left(6 l_{\mathrm{a}}\right)\right)^{1 / 3}\right)$ is the length scale of the brush height.

because histone proteins are desorbed from DNA chains when RNAP collides with nucleosomes during transcription; this recruits more RNAP in the regions. Because the number of RNAP in the system is fixed, RNAP is depleted in the other regions of the brush, and the transcription rate is small in those regions.

\section{DISCUSSION}

Our theory predicts that DNA brushes show phase separations when they are confined in a finite space and the region of twophase coexistent states have a critical point. The conditions of the critical point are sensitive to the rescaled rate constant $\eta_{0}$, the combination $n_{-}$of the second virial coefficients, and applied pressures $\Pi_{\text {app. }}$. The phase separations are driven by the instability arising from the fact that the transcription rate increases as the local packing density of nucleosomes decreases, and, in turn, the local packing density of nucleosomes decreases as the transcription rate increases. The excluded volume interactions between RNAP and nucleosomes are involved in both of the processes. During passive diffusion, RNAP is excluded from the brush region due to the excluded volume interactions between RNAP and nucleosomes. In contrast, during transcription, nucleosomes dissociate (namely, histone proteins are desorbed from DNA chains) when RNAP collides with nucleosomes. The same type of interactions end up with different results, depending on whether RNAP performs passive diffusion or active unidirectional motion.

Our theory predicts that DNA brushes show macroscopic phase separations in a confined space. Because two coexistent states are stable with different brush heights, the patterns of domains due to the phase separations may be sensitive to the stiffness of the surfaces, to which DNA chains are end-grafted. For the cases that the surfaces are very stiff, the distance between the two end-grafting surfaces are fixed. With this constraint, DNA chains may show microphase separations, where the space above domains of the collapsed state is filled by DNA chains in the swollen state, analogous to the dimple phase of binary polymer brushes. ${ }^{28-30}$ By contrast, for the cases that the surfaces of DNA brushes are flexible, the regions of two coexisting phases can separate in the lateral direction. Our theory is thus ideally tested by experiments that use DNA brushes on such surfaces. Experimentally, DNA brushes on soft surfaces may be prepared, for example, by end-grafting DNA chains on lipid membranes. ${ }^{31}$ DNA brushes may show various patterns when one changes the bending rigidity of the lipid membranes by protein decoration. ${ }^{32}$ This argument implies that the stiffness of nuclear membranes may play an important role in driving the phase separation of chromatin and organizing the pattern of domains.

In our theory, we have used the Maxwell construction to derive the binodal curves of the miscibility phase diagram (see Figure 5). However, the two coexisting phases in the region that is enclosed by the binodal curves are both nonequilibrium steady states, and thus the Maxwell construction may not be applicable. Although exactly determining the binodal curves is not the scope of our paper, it is of interest to discuss the validity of this treatment. Indeed, there are theories that use the Maxwell construction to locate the first order phase transitions between two nonequilibrium steady states. ${ }^{33-35}$ Hill and Chen have argued that the Maxwell construction is effective for the lattice models of interacting enzymes that take only two states. ${ }^{33}$ Indeed, our DNA chain segments take only two states (vacant or occupied by nucleosomes). However, their arguments are not directly applicable to our theory that treats only the mean values of the nucleosome occupancy and of the brush height. With our approximation, we do not take into account the contributions of the entropy production due to transcription under applied pressures (see eq 15), because the local concentration of RNAP in the solution of the brush region is very small; the work done by the applied pressures (which is used to predict the binodal curves) does not include this contribution. The steady state thermodynamics states that the minimum of the excess work (which does not include the entropy production to keep the system nonequilibrium) is the change of (the nonequilibrium version of) the Helmholtz free energy, analogous to the principle of minimum work. ${ }^{36}$ Our treatment is thus a good approximation at the vicinity of the 
binding transitions, where transcription rates are relatively small.

Equation 15 only has a unique solution for $\Pi_{\text {app }}>0$, at least, with a specific set of parameters that are used for our calculations (see also section S4 in the SI). The binding transitions that are predicted by our theory are thus different from the coil-globule transitions. Nevertheless, the cooperativity arising from the attractive interactions between nucleosomes is involved in the condensation of nucleosomes. The binding transitions of DNA brushes may be analogous to the gas-liquid phase transitions. It is thus of interest to discuss whether DNA brushes show phase separations, even when they are connected with reservoirs, where the number of RNAP and histone proteins is not fixed. However, the thermal fluctuations are not large enough to dissociate nucleosomes. Whether DNA brushes show phase separations in bulk solutions thus may depend on the fluctuations involved in the transcription dynamics. It is of interest to theoretically predict how the latter fluctuations are involved in the critical dynamics and phase separations of chromatin structures, and it will be the subject of our future study.

\section{CONCLUSION}

Our theory predicts that chromatin brushes show phase separations for the cases that these brushes are confined in a finite space. These phase separations are driven by the instability arising from the fact that the transcription rate increases as the local concentration of nucleosomes decreases due to the excluded volume interactions between nucleosomes and RNAP during passive diffusion and the local concentration of nucleosomes, in turn, decreases as the transcription rate increases due to the collision between RNAP and nucleosomes during transcription.

Our theory also predicts that chromatin brushes show critical points. The critical points are sensitive to the rate constants involved in transcription, the changes of the interactions between DNA chain segments by assembling nucleosomes, and applied pressures. In biological systems, the rate constants for transcription are modulated by transcription factor, and the attractive interactions between nucleosomes are modulated by histone modification. Indeed, transcription factors and histone modification are both thought to be important factors to maintain pluripotency of stem cells and also to drive differentiation. Moreover, recent experiments have shown that tension that is applied to nuclei by the cytoskeleton plays an important role in driving differentiation ${ }^{2}$ and determining the lineage. ${ }^{37}$ It is thus tempting to relate our theory to the phase separations of chromatin structures in the nuclei of stem cells during differentiation. Experimental tests of our theory on simple DNA brushes may provide insight in the relevance of the phase separations of chromatin brushes to the observed dynamics of chromatin structures in stem cells.

\section{ASSOCIATED CONTENT}

\section{S Supporting Information}

The Supporting Information is available free of charge on the ACS Publications website at DOI: 10.1021/acs.langmuir.6b00442.

Formal formulation of the theory, diffusion of RNAP in the solution, linear stability analysis, and coil-globule transitions (PDF)

\section{AUTHOR INFORMATION}

\section{Corresponding Author}

*E-mail: tyamamoto@nuap.nagoya-u.ac.jp.

\section{Notes}

The authors declare no competing financial interest.

\section{ACKNOWLEDGMENTS}

H.S. thanks the KITP at Santa Barbara for hospitality where part of this work has been performed and the National Science Foundation under Grant No. NSF PHY11-25915 for support. We are grateful to the reviewers for constructive comments.

\section{REFERENCES}

(1) Schiessel, H. The physics of Chromatin. J. Phys.: Condens. Matter 2003, 15, R699-R774.

(2) Talwar, S.; Kumar, A.; Rao, M.; Menon, G. I.; Shivashankar, G. V. Correlated Spatio-Temporal Fluctuations in Chromatin Compaction States Characterize Stem Cells. Biophys. J. 2013, 104, 553-564.

(3) Safran, S. A. Statistical Thermodynamics of Surfaces, Interfaces, and Membranes; Westview Press: Boulder, CO, 2003.

(4) Mühlbacher, F.; Holm, C.; Schiessel, H. Controlled DNA compaction within chromatin: The tail-bridging effect. Europhys. Lett. 2006, 73, 135-141.

(5) Widom, J. Physicochemical Studies of the Folding of the $100 \AA$ Nucleosome Filament into the 300 Å Filament: Cation Dependence. J. Mol. Biol. 1986, 190, 411-424.

(6) Mangenot, S.; Leforestier, A.; Vachette, P.; Durand, D.; Livolant, F. Salt-Induced Conformation and Interaction Changes of Nucleosome Core Particles. Biophys. J. 2002, 82, 345-356.

(7) Mangenot, S.; Raspaud, E.; Tribet, C.; Belloni, L.; Livolant, F. Interactions between isolated nucleosome core particles: A tailbridging effect? Eur. Phys. J. E 2002, 7, 221-231.

(8) Blossey, R.; Schiessel, H. The dynamics of the nucleosome: thermal effects, external forces and ATP. FEBS J. 2011, 278, 36193632 .

(9) Kulić, I. M.; Schiessel, H. Chromatin Dynamics: Nucleosomes go Mobile through Twist Defects. Phys. Rev. Lett. 2003, 91, 148103.

(10) Kulić, I. M.; Schiessel, H. Nucleosome repositioning via loop formation. Biophys. J. 2003, 84, 3197-3211.

(11) Bintu, L.; Kopaczynska, M.; Hodges, C.; Lubkowska, L.; Kashlev, M.; Bustamante, C. The elongation rate of RNA polymerase determines the fate of transcribed nucleosomes. Nat. Struct. Mol. Biol. 2011, 18, 1394-1399.

(12) ten Heggeler-Bordier, B.; Muller, S.; Monestier, M.; Wahli, W. An Immuno-electron Microscopical Analysis of Transcribing Multinucleosomal Templates: What Happens to the Histones? J. Mol. Biol. 2000, 299, 853-858.

(13) Daube, S. S.; Bracha, D.; Buxboim, A.; Bar-Ziv, R. H. Compartmentalization by directional gene expression. Proc. Natl. Acad. Sci. U. S. A. 2010, 107, 2836-2841.

(14) Buxboim, A.; Daube, S. S.; Bar-Ziv, R. Synthetic Gene Brushes: a Structure-Function Relationship. Mol. Syst. Biol. 2008, 4, 181.

(15) Bracha, D.; Karzbrun, E.; Daube, S. S.; Bar-Ziv, R. H. Emergent Properties of Dense DNA Phases toward Artificial Biosystems on a Surface. Acc. Chem. Res. 2014, 47, 1912-1921.

(16) Bracha, D.; Karzbrun, E.; Shemer, G.; Pincus, P. A.; Bar-Ziv, R. H. Entropy-driven collective interactions in DNA brushes on a biochip. Proc. Natl. Acad. Sci. U. S. A. 2013, 110, 4534-4538.

(17) Yamamoto, T.; Safran, S. A. Transcription rates in DNA brushes. Soft Matter 2015, 11, 3017-3021.

(18) Richmond, T. J.; Finch, J. T.; Rushton, B.; Rhodes, D.; Klug, A. Structure of the nucleosome core particle at $7 \AA$ resolution. Nature 1984, 311, 532-537.

(19) Luger, K.; Mäder, A. W.; Richmond, R. K.; Sargent, D. F.; Richmond, T. J. Crystal structure of the nucleosome core particle at $2.8 \AA$ A resolution. Nature 1997, 389, 251-260. 
(20) Marko, J. K.; Siggia, E. D. Stretching DNA. Macromolecules 1995, 28, 8759-8770.

(21) Alexander, S. Adsorption of chain molecules with a polar head: a scaling description. J. Phys. (Paris) 1977, 38, 983-987.

(22) Kaplan, N.; Moore, I. K.; Fondufe-Mittendorf, Y.; Gossett, A. J.; Tillo, D.; Field, Y.; LeProust, E. M.; Hughes, T. R.; Lieb, J. D.; Widom, J.; Segal, E. The DNA-encoded nucleosome organization of a eukaryotic genome. Nature 2009, 458, 362-366.

(23) Nicolai, T.; Mandel, M. Ionic Strength Dependence of the Second Virial Coefficient of Low Molar Mass DNA Fragments in Aqueous Solutions. Macromolecules 1989, 22, 438-444.

(24) Mazurkiewicz, J.; Kepert, J. F.; Rippe, K. On the Mechanism of Nucleosome Assembly by Histone Chaperone NAP1. J. Biol. Chem. 2006, 281, 16462-16472.

(25) Mollazadeh-Beidokhti, L.; Mohammad-Rafiee, F.; Schiessel, H. Active Nucleosome Displacement: A Theoretical Approach. Biophys. J. 2009, 96, 4387-4398.

(26) Marko, J. F.; Witten, T. A. Phase separation in a Grafted Polymer Layer. Phys. Rev. Lett. 1991, 66, 1541-1544.

(27) Komura, S.; Safran, S. A. Scaling theory of mixed amphiphile monolayers. Eur. Phys. J. E: Soft Matter Biol. Phys. 2001, 5, 337-351.

(28) Müller, M. Phase diagram of a mixed polymer brush. Phys. Rev. E: Stat. Phys., Plasmas, Fluids, Relat. Interdiscip. Top. 2002, 65, 030802. (29) Minko, S.; Müller, M.; Usov, D.; Scholl, A.; Froeck, C.; Stamm, M. Lateral versus Perpendicular Segregation in Mixed Polymer Brushes. Phys. Rev. Lett. 2002, 88, 035502.

(30) van Lehn, R. C.; Alexander-Katz, A. Lateral phase separation of mixed polymer brushes physisorbed on planar substrates. J. Chem. Phys. 2011, 135, 141106.

(31) Nikolov, V.; Lipowsky, R.; Dimova, R. Behavior of Giant Vesicles with Anchored DNA Molecules. Biophys. J. 2007, 92, 43564368.

(32) Tsai, F. C.; Koenderink, G. H. Shape control of lipid bilayer membranes by confined actin bundles. Soft Matter 2015, 11, 88348847.

(33) Hill, T. L.; Chen, Y. D. Interacting enzyme systems at steady state: Location of the phase transition in approximations of the mean field type. Proc. Natl. Acad. Sci. U. S. A. 1978, 75, 3015-3018.

(34) Keizer, J. Maxwell-type construcions for multiple nonequilibrium steady states. Proc. Natl. Acad. Sci. U. S. A. 1978, 75, 3023-3026.

(35) Ge, H.; Qian, H. Thermodynamic Limit of a Nonequilibrium Steady State: Maxwell-Type Construction for a Bistable Biochemical System. Phys. Rev. Lett. 2009, 103, 148103.

(36) Hatano, T.; Sasa, S. Steady-State Thermodynamics of Langevin Systems. Phys. Rev. Lett. 2001, 86, 3463-3466.

(37) Engler, A. J.; Sen, S.; Sweeney, H. L.; Discher, D. E. Matrix elasticity directs stem cell lineage specification. Cell 2006, 126, 677689. 